REDES- Revista hispana para el análisis de redes sociales.

Vol.3,\#4, sept-nov. 2002.

http://revista-redes.rediris.es

\title{
Las comunidades locales como estructuras meso ${ }^{1}$.
}

\author{
A. Ferrand, Catedrático en Sociología \\ Université des Sciences et Technologies de Lille (Francia)
}

\begin{abstract}
In between micro and macro levels : meso. If macro is defined by national, state or market regulation, and micro by relational interpersonnal regulation, a major agency of intermediate regulation has been defined as "local communities" (Wellmann, Fischer). Apart the organizational (local organizations, clubs, shops...) and institutional (local power, parties,...) dimensions of localities, it is possible to define some pertinent structural properties of systems of private interpersonal relations : one is the degree of local closure (Barnes: local vs transversal networks), or the "duality" of these systems. Grounded on the idea that the efficiency of "bridges" rest upon the kind of cliques they connect (Lin), it is possible to define various types of micro-structures, the distributions of which in different localities are proxy indicators of the different meso structures of relational systems as combinations of "local" and "unlocal" networks.
\end{abstract}

\section{Resumen}

Entre el nivel micro y el nivel macro: meso. Si el nivel macro está definido por la regulación nacional, estatal o del mercado y el nivel micro por la regulación interpersonal, una agencia de regulación intermediaria de mayor importancia ha sido definida como "comunidad local" (Wellman, Fischer). Aparte de las dimensiones organizacionales (organizaciones locales, clubes, tiendas...) e institucionales (poder local, partidos...) locales, es posible definir algunas propiedades estructurales pertinentes de los sistemas de relaciones interpersonales privadas: uno es el grado de "cerrado" (closure) local (Barnes: redes locales vs. transversales), o la "dualidad" de estos sistemas. Apoyándose en la idea de que la eficiencia de los "puentes" reside en el tipo de cliques que conectan (Lin), es posible definir tipos variados de micro-estructuras, cuyas distribuciones en diferentes localidades son indicadores sucedáneos o aproximaciones de las diferentes meso estructuras de sistemas relacionales como combinaciones de redes "locales" y "no locales".

\section{Un postulado: la existencia de "sistemas locales de salud"}

La regulación de los sistemas de salud es una cuestión importante en muchos países, sin embargo, la definición de lo que es un "sistema de salud" es variada. Nuestro grupo de investigación trabaja con el postulado de que los "sistemas locales de salud" existen en

\footnotetext{
${ }^{1}$ Comunicación presentada en la Conferencia Temática Europea para Analistas de Redes Sociales, LINET: "La relación Micro-Macro", Lille (Francia), Mayo 30-31, 2002, traducida por Ainhoa de Federico.
} 
tanto que sistemas que pueden diferir de un lugar - utilizando un término vago- a otro 2. La cuestión que nos interesa no es la clásica descripción geográfica de las provisiones diferenciales de servicios de salud en áreas diferentes, ni las tasas de mortalidad, o cuestiones similares. Lo que nos interesa son los sistemas locales de salud en tanto que sistemas caracterizados por estructuras sociales diferentes.

\section{Órdenes institucionales diferentes}

Las instituciones, organizaciones, grupos voluntarios etc. instalados y ofreciendo servicios en una localidad son componentes de los sistemas locales de salud. De acuerdo con la argumentación de Fischer sobre el papel de las instituciones locales especializadas que sustentan la emergencia de subculturas (Fischer,1982), es posible analizar cómo la naturaleza, la diversidad de estas instituciones, y el tipo de redes inter-organizacionales que crean, producen especificidades locales en las pautas normativas que guían los comportamientos de profesionales y profanos, y la gestión de la salud pública. Estas redes inter-organizacionales forman parte del orden institucional de la localidad.

\section{Diversas redes de discusión sobre salud}

Otro componente de estos sistemas locales de salud es el sistema relacional en el que las personas reciben y discuten ya sea información general sobre las enfermedades y sus tratamientos, ya información específica sobre los recursos locales de cuidados sanitarios, la calidad de los hospitales y de los profesionales de la salud ${ }^{3}$. Ese sistema relacional produce y transforma las representaciones, normas y saberes sobre la salud y los tratamientos, los juicios sobre la calidad de los servicios de salud y la difusión de las reputaciones que regulan el "mercado". También contribuye al control social (Ferrand, 2000). Por lo tanto es necesario definir y observar estos sistemas relacionales, identificar sus diferentes propiedades estructurales y descubrir cómo distintas propiedades estructurales producen resultados diversos.

\section{Propiedades estructurales de las redes de discusión sobre salud}

\footnotetext{
2 Esta presentación es un resultado de una investigación financiada por el programa del CNRS « SANTÉ-SOCIETE» y conducida por Geneviève CRESSON, Alexis FERRAND, Philippe LARDÉ, en el laboratorio del CNRS «Centre Lillois d'études et de recherches sociologiques et économiques » CLERSE.

${ }^{3}$ He basado este análisis en la misma perspectiva sobre las redes de discusión tal como la utilizada para estudiar el control normativo de la sexualidad (Ferrand, Mounier, 1994,1996; para una presentación más teórica y resultados finales ver 1998).
} 
A partir de este punto, la cuestión es decidir en qué medida pueden éstas redes de discusión ser comprendidas en tanto que sistemas relacionales y en tanto que organizaciones sociales específicas entre las limitaciones globales universalistas del "Sistema Nacional de Salud" y los intercambios interpersonales de información y de apoyo extremamente particularistas. En otras palabras, ¿en qué medida deberíamos definir un sistema relacional con sus propias propiedades estructurales y sus efectos funcionales al nivel meso?

Basándose en la teoría del capital social, es posible insistir en las variaciones de las propiedades estructurales de las redes de discusión en diferentes localidades; por ejemplo, características estructurales que permiten la circulación de información entre "profanos" y "especialistas". Los "especialistas" son personas que trabajan en el campo de la salud: forman círculos sociales - redes- que están más o menos ligados o abiertos a otras categorías de la población. Los especialistas son también personas que tienen una experiencia profunda o duradera sobre las enfermedades y sus tratamientos: ¿en qué medida comparten su saber en diversos círculos sociales - o por el contrario- la conservan en el pequeño ámbito de los íntimos? Hemos examinado tales propiedades estructurales comparando dos localidades (Cresson, Ferrand, Lardé, 2001).

Esta perspectiva es bastante clásica: es heredera de la tradición de los estudios de comunidades (Community Studies) aunque su metodología haya sido modernizada. EI postulado básico de este paradigma es que los sistemas locales existen y que debemos descubrir sus modelos formales y funcionales. Pero tal postulado no puede ser asumido para todos los componentes de las localidades.

Los datos empíricos muestran que las personas mantienen redes de discusión que incluyen mayor o menor número y proporción de personas viviendo "en la misma localidad" al igual que "en otra parte". . Estas pruebas sugieren que los sistemas relacionales locales están más o menos abiertos a los contactos exteriores. Esta formulación no cambia la asunción básica que se refiere a la consistencia del sistema local. Es posible proponer otra formulación, más compleja, pero con mayor capacidad heurística. Para introducirla, volvamos a los análisis de Barnes.

\section{Las aportaciones de Barnes: el sistema social local/ no local}

Barnes describió Bremnes en términos de un orden territorial e industrial - dos campos de orden institucional - , pero también insistió en cómo la estructura global de las redes de amistad y de parentesco - el "tercer campo" y las redes personales que lo componen -

\footnotetext{
${ }^{4}$ Era una idea básica de B. Wellman cuando analizó East York en los años ochenta (1979, 1982, 1988).
} 
están en la intersección de varios círculos formales y pueden ser pensados como redes sin fronteras.

“El tercer campo social no tiene unidades o fronteras; no tiene organización coordinadora. Está formado por los lazos con amigos y conocidos que cualquiera que haya crecido en la sociedad de Bremnes hereda parcialmente y sobre todo crea para sí mismo. [...] Para nuestro presente objetivo, sin embargo, quiero considerar la parte de la red total que queda cuando eliminamos los grupos y cadenas de interacción que pertenecen estrictamente a los sistemas territoriales e industriales. En la sociedad de Bremnes, lo que queda es mayoritariamente, aunque no exclusivamente, una red de lazos de parentesco, amistad, y vecindad. Esta red atraviesa la sociedad en su totalidad y no se detiene en las fronteras parroquiales. Relaciona gente de Bremnes con sus parientes y amigos en otras parroquias al igual que los entreteje dentro de la parroquia. Una red de este tipo no tiene fronteras externas, ni tiene ninguna división interna clara..." (Barnes, 1954, p.237)

Recordemos dos ideas: 1) podemos pensar en un sistema social en tanto que formado por elementos locales y no locales - los tres "campos" en términos de Barnes - cada uno de ellos con diferentes propiedades estructurales y con diferentes funciones. 2) podemos pensar en uno de esos campo en tanto que red ilimitada con fuertes propiedades “locales" y efectos locales importantes en la producción de representaciones sociales del estatus, la jerarquía y el consenso - es el objetivo de Barnes demostrar cómo el "tercer campo" es un elemento crucial de la regulación política y social del sistema global.

Entonces la cuestión ya no es la "apertura" de un sistema local existente a priori. Necesitamos nuevas formulaciones: podemos utilizar la idea de la "dualidad" de tal sistema que debería ser definido como local y simultáneamente no local.

\section{Un puente en el sistema total: tres elementos}

La dualidad local/no local puede ser formulada en términos estructurales con el concepto de puente. Un puente es una relación cuyos extremos pertenecen a dos sub-redes diferentes y no relacionadas. El puente, como tal, no es ninguna de las dos sub-redes. Pero la estructura de cada sub-red no puede ser caracterizada sin el puente, y las propiedades estructurales del puente no pueden ser definidas sin las propiedades de ambas sub-redes que relaciona (por ejemplo Lin, 2001). Este tipo de realidad tiene que ser conceptualizado como el sistema total formado por los tres elementos "sub-red A", "sub-red B", y el puente; o "estructura A", "estructura B" y "tipo de puente". De la misma manera, la definición de la dualidad implica simultáneamente una estructura interna, las propiedades estructurales de las posiciones donde se conecta el puente y la calidad del puente mismo. El marco de análisis debe tener en cuenta el sistema total formado por los elementos locales y no locales. 


\section{“Dualidad": intersección entre unidades locales y no locales}

Las personas que viven en diferentes localidades ${ }^{5}$ están conectadas con personas que viven en ellas y con personas que viven en otros lugares. Las localidades, los distintos "aquís", existen en tanto que órdenes institucionales colectivos (son territorios administrativos y políticos, comprenden agencias locales específicas, grupos voluntarios etcétera), y en tanto que contextos sociales diferenciados que ofrecen diversas oportunidades de encuentros (composición de la población segregación residencial, etc.). Pero en el marco del análisis propuesto, debemos definir la intersección entre la organización de los grupos e instituciones territorialmente limitados y los sistemas relacionales ilimitados; o cómo las localidades pueden ser caracterizadas por sus "formas de dualidad", es decir por sus formas de conectividad [interna-externa]. Podemos construir tipos formales con una oposición simplificada:

\begin{tabular}{l|l|l|l|}
\multicolumn{1}{c|}{$\begin{array}{c}\text { Formas de } \\
\text { dualidad }\end{array}$} & \multicolumn{2}{l}{$\begin{array}{l}\text { Conectividad } \\
\text { externa }\end{array}$} \\
\hline \multirow{3}{*}{$\begin{array}{l}\text { Conectividad } \\
\text { interna }\end{array}$} & Fuerte & & \\
\cline { 2 - 4 } & Débil & & \\
\cline { 2 - 4 } & &
\end{tabular}

\section{Dificultades del análisis empírico}

Si estamos interesados en los procesos sociales que tienen lugar en círculos limitados de las elites o grupos profesionales especializados, es posible examinar la red total de todos los actores (por ejemplo Laumann \& Papi, 1976; Galaskiewitz, 1985). Pero si estamos interesados en los procesos que ocurren en la población "global" de las localidades, sólo podemos utilizar encuestas de redes personales: las proporciones de los diversos tipos de redes personales en una población dada pueden ser utilizados como indicadores de la estructura relacional subyacente desconocida que relaciona esta población (Ferrand 1997).

Los partidarios de aproximaciones estructurales formales del análisis de redes pueden criticar la debilidad de tales indicadores, pero permítanme añadir que, por el contrario es

\footnotetext{
${ }^{5}$ Algunos lectores pueden considerar este énfasis en la localidad como deliciosamente coloreado con tintes de los años sesenta. Les recuerdo tres hechos: a) incluso si las personas pueden buscar información en internet, incluso si la enfermedad tiene una fuerte dimensión simbólica e imaginaria, los problemas de salud son muy concretos y limitan a las personas a buscar apoyos sociales fuertes y eficientes de forma práctica, a menudo locales b) uno de los factores más importantes para escoger un médico es que viva cerca; c) el fracaso de la macro regulación de los costes curativos de la salud, la necesidad de desarrollar la prevención, el intento de racionalizar el cuidado de la salud mediante la cooperación entre profesionales de la salud han inducido, en Francia, un fuerte compromiso político en el desarrollo de políticas sanitarias locales.
} 
fácilmente identificable la incapacidad de las aproximaciones de redes totales para dar cuenta de cualquier proceso colectivo al nivel meso cuando éste no puede ser expresado a través de las relaciones entre un número limitado de actores - a menudo colectivos identificables y rastreables. Por otra parte, el análisis de redes sociales para redes completas está evolucionando de, volviendo a la distinción efectuada por C.Levi-Strauss, una aproximación "mecánica" a una "estadística". La aproximación mecánica entiende los datos, exhaustivamente completos, como la verdadera expresión de un orden social prescriptivo, mientras que la perspectiva estadística considera los datos como una expresión aproximada de un orden social basado en preferencias individuales limitadas por reglas proscriptivas. La descripción de las propiedades de una red total mediante el censo de tríadas, y ahora la evaluación estadística de estas distribuciones mediante el modelo p*, abren nuevas avenidas a la formalización de las estructuras de las redes mediante distribuciones estadísticas diferenciales de sub-estructuras típicas (en díadas, tríadas, etc...). Cuando estemos familiarizados con tales descripciones, estaremos más fácilmente de acuerdo con que a) podemos pensar en las estructuras en tanto que modelos probabilísticos; b) las distribuciones de sub-estructuras típicas a partir de muestras aleatorias ofrecen indicadores empíricos suficientes sobre los modelos de las estructuras de los sistemas meso de los que nos estamos ocupando.

\section{I lustración empírica}

Hemos observado redes personales de discusión sobre salud y tenemos datos sobre:

- dos categorías de relaciones 1) entre ego (por definición un actor local) y diferentes alters que viven en la misma localidad (relaciones locales etiquetadas mediante "L"); 2) entre ego y alters que viven fuera de la localidad (relaciones no locales etiquetadas mediante "U");

- diferentes composiciones de redes personales que combinan relaciones locales (L) y relaciones no locales (U).

Las combinaciones variadas pueden ser simplificadas para llegar a un número limitado de tipos de redes personales pertinentes que deberían ser comprendidas como tipos de micro estructuras. La Tabla 1 muestra, en la columna de la izquierda, los modelos de composición de las redes de confidentes a propósito de cuestiones de salud. Cada letra representa una relación "L" para cada relación local, o "U" para cada relación no local. En la columna central se encuentran la frecuencia y el porcentaje en la muestra total de cada modelo. He agrupado los diferentes modelos (varias líneas) en tipos a priori de micro-estructuras. La columna de la derecha de la tabla indica las etiquetas y las frecuencias globales para cada tipo de micro-estructura. 
Tabla 1: De los tipos de composiciones de redes personales a los tipos de microestructuras

\begin{tabular}{|c|c|c|c|c|c|}
\hline \multicolumn{2}{|c|}{$\begin{array}{c}\text { Composición de las } \\
\text { redes } \\
\text { (Local / No local) }\end{array}$} & $\mathbf{N}$ & $\%$ & \multicolumn{2}{|c|}{$\begin{array}{l}\text { Tipo de micro-estructura } \\
\text { relacional }\end{array}$} \\
\hline \multicolumn{2}{|c|}{ Sin relación } & 92 & 17.79 & CENSURADA & $17.8 \%$ \\
\hline \multicolumn{2}{|l|}{$\mathrm{L}$} & 162 & 31.33 & Dí ADA AI SLADA & $31.3 \%$ \\
\hline \multicolumn{2}{|l|}{$\mathrm{LL}$} & 78 & 15.09 & LI MI TADA & $24.0 \%$ \\
\hline \multicolumn{2}{|l|}{ LLL } & 35 & 6.77 & LOCALMENTE & \\
\hline \multicolumn{2}{|l|}{ LLLL } & 7 & 1.35 & & \\
\hline \multicolumn{2}{|l|}{ LLLLL } & 3 & 0.58 & & \\
\hline \multicolumn{2}{|c|}{ LLLLLL } & 1 & 0.19 & & \\
\hline \multicolumn{2}{|c|}{ LLLLLL } & 16 & 3.09 & DIFUSOR & $4.5 \%$ \\
\hline LLL & U & 4 & 0.77 & & \\
\hline LLL & UU & 1 & 0.19 & \multirow{2}{*}{\multicolumn{2}{|c|}{ Más relaciones locales que no }} \\
\hline \multirow{2}{*}{$\frac{\text { LLLL }}{\mathrm{L}}$} & $U$ & 2 & 0.39 & & \\
\hline & $U$ & 31 & 6.00 & \multicolumn{2}{|l|}{ COSMOPOLITA } \\
\hline L & UU & 14 & 2.71 & & \\
\hline $\mathrm{L}$ & UUU & 3 & 0.58 & & \\
\hline $\mathrm{L}$ & UUUU & 2 & 0.39 & \multirow{2}{*}{\multicolumn{2}{|c|}{$\begin{array}{l}\text { Menor o igual número de } \\
\text { relaciones locales que no locales }\end{array}$}} \\
\hline LL & UU & 5 & 0.97 & & \\
\hline \multirow{5}{*}{ LLL } & UUU & 1 & 0.19 & & \\
\hline & $\bar{U}$ & 34 & 6.58 & NO LOCAL & $11.6 \%$ \\
\hline & UU & 17 & 3.29 & & \\
\hline & UUU & 4 & 0.77 & & \\
\hline & UUUU & 5 & 0.97 & & \\
\hline
\end{tabular}

No es mi intención discutir en profundidad la racionalidad de agrupar los modelos en tipos de micro-estructuras. La idea de conjunto es considerar las diferentes potencialidades de las redes para articular las sub-redes locales y no locales, es decir, si conectan muchos o pocos lazos locales a muchos o pocos lazos no locales. Podemos identificar tres tipos de micro estructuras locales (en lo alto de la tabla): a) las estructuras "censuradas": los actores que no poseen ningún confidente; b) las "díadas aisladas": relaciones entre dos personas locales; c) estructuras "localmente limitadas": tres o más actores que pertenecen a la localidad.

- un tipo de micro-estructura no local (abajo de la tabla): un actor focal con todos sus asociados fuera de la localidad;

- dos tipos de micro-estructuras local/no local (centro de la tabla): a) "difusores" es decir, estructuras en que la mayoría de las relaciones son locales; b) "cosmopolitas" es decir estructuras compuestas por un número igual o mayor de no locales que de locales. Ambos tipos contribuyen a conectar la vida social local con campos relacionales externos. El primero, debido a una proporción mayor de relaciones locales, es probablemente más capaz de difundir localmente los recursos externos y la información; el segundo, debido a su mayor proporción de relaciones externas, es probablemente más capaz de capturar más recursos y más variados en la red no local. 
Estoy de acuerdo en que se podrían proponer otras agrupaciones, pero en cualquier caso de lo que se trata aquí es de identificar las diversas propiedades de "puenteo" de las micro-estructuras. Los datos muestran que una propiedad básica de la red es estar poco entretejida, el $17.8 \%$ de los habitantes no tienen relaciones de discusión sobre salud y el $31.3 \%$ pertenecen a una díada local aislada. Sólo una pequeña proporción de las micro estructuras $(15.3 \%=4.5+10.8 \%)$ tienen la propiedad de combinar relaciones locales y no locales. Existe un tipo de estructura muy interesante que es totalmente no local (11.6\%): una persona que vive "aquí" pero que está exclusivamente conectado fuera de la localidad.

\section{Formas de dualidad}

Ahora es posible caracterizar diversas formas de dualidad mediante las distribuciones de estos tipos. La cuestión clave es que estas distribuciones dan información no sobre las "localidades" en si mismas, sino sobre las maneras en que unidades locales efectúan la intersección con sistemas relacionales no locales ilimitados. Si asumimos que el sistema "real" está formado a partir de las intersecciones entre unidades locales y sistemas relacionales no locales, estas distribuciones capturan una imagen parcial de ese sistema. Esa imagen está definida, conformada a partir de un "punto de vista": está observada a partir de las unidades residenciales locales, pero mira simultáneamente lo que es local y no local en las localidades.

Para las dos localidades en que la investigación fue llevada a cabo, Lens y Tourcoing, la tabla 2 muestra las distribuciones de los diferentes tipos de micro-estructuras.

Tabla 2: Distribuciones de micro-estructuras como formas diferenciales de dualidad en dos localidades

\begin{tabular}{l|c|c|c}
\multicolumn{1}{c|}{$\begin{array}{c}\text { TI POS DE MI CRO- } \\
\text { ESTRUCTURAS }\end{array}$} & LENS & TOURCOI NG & TOTAL \\
\hline CENSURADAS & 61 & 31 & 92 \\
Sin relación & $24 \%$ & $12 \%$ & $17.8 \%$ \\
\hline Dí ADAS AI SLADAS & 92 & 70 & 162 \\
1 relación local & $35 \%$ & $27 \%$ & $31.3 \%$ \\
\hline LI MI TADA LOCALMENTE & 47 & 77 & 124 \\
2 o más relaciones locales & $18 \%$ & $30 \%$ & $24.0 \%$ \\
\hline DI FUSOR & 6 & 17 & 23 \\
Más relaciones locales que & $2 \%$ & $7 \%$ & $4.5 \%$ \\
no locales & & & \\
\hline COSMOPOLI TA & 14 & $42 \%$ & $10.8 \%$ \\
Menos (o las mismas) & $5 \%$ & $16 \%$ & 60 \\
relaciones locales & 41 & 19 & $11.6 \%$ \\
\hline NO LOCAL & $16 \%$ & $8 \%$ & \\
Sólo relaciones no locales & & & \\
\hline
\end{tabular}




\section{TOTAL}

\begin{tabular}{c|c|c}
261 & 256 & 517 \\
$100 \%$ & $100 \%$ & $100 \%$
\end{tabular}

La comparación de estas dos distribuciones indica dos tipos muy diferentes de intersección entre las unidades locales y no locales.

- Las intersecciones entre micro estructuras (difusores + cosmopolitas) representan 7\% $(=2 \%+5 \%)$ en Lens y $23 \%(=7 \%+16 \%)$, es decir, tres veces más, en Tourcoing.

- Las estructuras no locales representan más o menos 16\% en Lens, $8 \%$ en Tourcoing, es decir dos veces menos.

- El aislamiento, es decir la ausencia de relaciones y las díadas locales representan 59\% en Lens, y $39 \%$ en Tourcoing.

Si volvemos a la tipología simplificada de las formas de dualidad, podemos utilizar estos ejemplos empíricos:

\begin{tabular}{ll|l|l|}
\multicolumn{1}{c|}{$\begin{array}{c}\text { Formas de } \\
\text { dualidad }\end{array}$} & \multicolumn{2}{l}{$\begin{array}{l}\text { Conectividad } \\
\text { externa }\end{array}$} \\
\hline \multirow{3}{*}{$\begin{array}{l}\text { Conectividad } \\
\text { interna }\end{array}$} & Fuerte & Turcoing & \\
\cline { 2 - 4 } & Débil & & Lens \\
\cline { 2 - 4 } & &
\end{tabular}

Lens presenta una estructura débilmente tejida con pocas conexiones exteriores, mientras que Tourcoing presenta la imagen inversa: una estructura densamente tejida con fuertes conexiones exteriores.

\section{Conclusiones}

a) Este artículo es un intento de definir "formas de dualidad" como un tipo de propiedades estructurales de las localidades.

b) Sugiere que la distribución de los tipos de micro- estructuras pueden ser utilizados como indicadores sucedáneos o aproximaciones disponible de estas propiedades estructurales.

c) En este ejemplo, hay una asimetría de información: sabemos dónde están los lazos locales, pero no sabemos a dónde van los lazos no locales.

d) Esa debilidad debe ser evaluada en relación a la capacidad heurística de la aproximación propuesta. El fuerte contraste entre las dos distribuciones presentadas en la tabla 2 es suficiente para identificar diversas formas de dualidad. 
e) Una investigación más amplia debería incluir más de dos localidades para mostrar cómo estas formas de dualidad pueden ser uno de los factores que permitan explicar los procesos de difusión de información y de la formación de representaciones y juicios sociales.

f) Sugiere que una localidad no es per se una realidad de nivel meso. Debemos pensar las localidades como elementos de un sistema meso mayor en el que las unidades locales y no locales se encuentran en intersección. La teoría debe tratar tanto con la organización local de las relaciones como con la red ilimitada, compleja y difícil de rastrear de relaciones no locales. Una localidad no es un sistema "local" más o menos abierto, es un sistema meso, es la intersección específica entre unidades locales y no locales.

\section{Bibliografía}

Barnes J.A (1954). "Class and committees in a Norwegian Island parish", Human Relations, 7 (9-58).

Cresson G. Ferrand A. Lardé P. (2001). Les systèmes locaux de santé. Programme CNRS «Santé Société », Lille CNRS Clersé.

Ferrand A., Mounier L. (1994). "Social discourse and normative influences..." Sexual behaviour and AIDS. A. Spira, N. Bajos, and the ACSF Group. Aldershot, Avebury (140148).

Ferrand A. Mounier L. (1996). "Talking about sexuality: An analysis of relations between confidants". Sexuality and the social sciences. M. Bozon et H. Leridon Eds. Aldershot, Dartmouth (265-288).

Ferrand, A. (1997). «La structure des systèmes de relations » L'Année Sociologique 1997, 47(1) (37-54).

Ferrand A., Mounier L. (1998). "L'influence des réseaux de confidence sur les relations sexuelles", Bajos N., Bozon M., Ferrand A., Giami A., Spira A. (eds) La sexualité aux temps du Sida. Paris: PUF (255-304)

Ferrand A (2000). "Effets des structures des réseaux de discussion sur la production des réputations", en Cresson G. et Schweyer F.X., (eds.), Les usagers du système de soins. Rennes: Editions de I'ENSP.

Galaskiewicz J. (1985). Social organization of the urban grant economy: a study of business philanthropy and non profit organizations. New York: Academic Press.

Laumann E.O. Pappi F.U. (1976). Networks of collective action. A perspective on community influence systems. New York: Academic Press.

Lin N. (2001). Social capital: a theory of social structure and action. Cambridge: University Press. 
Wasserman S. Faust K. (1994). Social Network analysis. Cambridge: Cambridge University Press.

Wellman B. (1979). "The community question: The intimate networks of east yorkers", American J ournal of Sociology, vol.84, (5).

Wellman B. (1982). "Studying personal communities". Marsden P. Lin N. (eds.), Social structure and network analysis. Beverly Hills: Sage.

Wellman B. Berkowitz S.D. (eds.). (1988). Social Structures. A Network Approach. Cambridge: Cambridge University Press. 